\title{
The Sylow p-Subgroups of Tame Kernels in Dihedral Extensions of Number Fields \\ by
}

\author{
Qianqian CUI and Haiyan ZHOU
}

Presented by Andrzej SKOWROŃSKI

Summary. Let $F / E$ be a Galois extension of number fields with Galois group $D_{2^{n}}$. In this paper, we give some expressions for the order of the Sylow $p$-subgroups of tame kernels of $F$ and some of its subfields containing $E$, where $p$ is an odd prime. As applications, we give some results about the order of the Sylow $p$-subgroups when $F / E$ is a Galois extension of number fields with Galois group $D_{16}$.

1. Introduction. Let $F$ be a number field, $\mathcal{O}_{F}$ the ring of integers in $F$, and $K_{2}(F)$ the Milnor $K$-group of $F$. The tame symbol on $F$ induces, for each finite prime ideal $\mathfrak{p}$, a homomorphism

$$
\tau_{\mathfrak{p}}: K_{2}(F) \rightarrow k_{\mathfrak{p}}^{*}
$$

defined by

$$
\tau_{\mathfrak{p}}\{a, b\} \equiv(-1)^{\nu_{\mathfrak{p}}(a) \nu_{\mathfrak{p}}(b)} \frac{a^{\nu_{\mathfrak{p}}(b)}}{b^{\nu_{\mathfrak{p}}(a)}}(\bmod \mathfrak{p}),
$$

where $\nu_{\mathfrak{p}}$ denotes the $\mathfrak{p}$-adic valuation. The tame kernel of $F$ is the kernel of $\tau$, where

$$
\tau=\bigoplus \tau_{\mathfrak{p}}: K_{2}(F) \rightarrow \bigoplus_{\mathfrak{p} \text { finite }} k_{\mathfrak{p}}^{*}
$$

In 1973, Quillen [6] proved that the $K$-group $K_{2}\left(\mathcal{O}_{F}\right)$ coincides with the tame kernel, and $K_{2}\left(\mathcal{O}_{F}\right)$ is finite.

There are many results describing the structure of the tame kernels of algebraic number fields and relating them to the class numbers of appropriate fields. The 2-primary part of the tame kernel $K_{2}\left(\mathcal{O}_{F}\right)$ for number fields 
$F$ has been intensively studied (see [3, [6]-8]). Furthermore, there are also some results concerning the $p$-primary part of the tame kernel when $p$ is odd (see [2], 44, [12]-[14]). Let $F / E$ be a Galois extension of number fields with Galois group $D_{2^{n}}$. The second author [12] obtained some results on tame kernels in the case $n=3$, i.e., $\operatorname{Gal}(F / E)=D_{8}$.

In this paper, we prove some expressions for the order of the Sylow $p$-subgroups of tame kernels of $F$ and some of its subfields containing $E$ for any integer $n \geq 3$. As applications, in Section 3, we give some results about the order of the Sylow $p$-subgroups when $F / E$ is a Galois extension of number fields with Galois group $D_{16}$.

2. Main results. Throughout the paper we use the following notation:

- $D_{2^{n}}$ is the dihedral group of order $2^{n}$, i.e., $D_{2^{n}}=\langle\sigma, \tau| \sigma^{2^{n-1}}=1$, $\left.\tau^{2}=1, \tau \sigma \tau^{-1}=\sigma^{-1}\right\rangle$.

- $E^{m} / E$ is a finite extension of number fields of degree $m$.

- $A(p)$ denotes the Sylow $p$-subgroup of a finite group $A$.

- $|A|$ denotes the order of a finite group $A$.

- $x={ }_{p} y$ means $v_{p}(x)=v_{p}(y)$, where $x, y \in \mathbb{Z}$.

- $C_{m}$ is a cyclic group of order $m$.

- $V_{4}$ is Klein's four group.

Now, we start with some well-known facts which will be the basis of this paper.

Let $F / E$ be a finite extension of number fields. In algebraic $K$-theory, a transfer $\operatorname{tr}_{F / E}$ is defined which is a group homomorphism

$$
\operatorname{tr}_{F / E}: K_{2}(F) \rightarrow K_{2}(E) \text {. }
$$

Denote by $K_{2}(F / E)$ the kernel of the map $\operatorname{tr}_{F / E}: K_{2}\left(\mathcal{O}_{F}\right) \rightarrow K_{2}\left(\mathcal{O}_{E}\right)$. Obviously, the Sylow $p$-subgroup $K_{2}(F / E)(p)$ of $K_{2}(F / E)$ is the kernel of the $\operatorname{map} \operatorname{tr}_{F / E}: K_{2}\left(\mathcal{O}_{F}\right)(p) \rightarrow K_{2}\left(\mathcal{O}_{E}\right)(p)$.

Lemma 1. For every prime $p \nmid(F: E)$,

$$
K_{2}\left(\mathcal{O}_{F}\right)(p) \cong K_{2}(F / E)(p) \times K_{2}\left(\mathcal{O}_{E}\right)(p) .
$$

LEMma 2. If $L$ is an intermediate field of $F / E$, then

$$
\operatorname{tr}_{F / E}=\operatorname{tr}_{L / E} \circ \operatorname{tr}_{F / L} .
$$

Lemma 3. If $F / E$ is a Galois extension with Galois group $G$, then for every prime $p \nmid(F: E)$, the homomorphism $j: K_{2}\left(\mathcal{O}_{E}\right)(p) \rightarrow K_{2}\left(\mathcal{O}_{F}\right)(p)$ induced by $E \subset F$ is injective, and the transfer $\operatorname{tr}_{F / E}: K_{2}\left(\mathcal{O}_{F}\right)(p) \rightarrow$ $K_{2}\left(\mathcal{O}_{E}\right)(p)$ is surjective. Moreover, $j \circ \operatorname{tr}_{F / E}=N_{F / E}$, where $N_{F / E}(x)=$ $\prod_{\sigma \in G} \sigma(x)$. 
Lemma 4 ([12, Theorem 1]). Let $E^{4} / E$ be a Galois extension with Galois group $V_{4}=\{1, a, b, a b\}, E_{a}^{2}$ the fixed field of $\langle a\rangle, E_{b}^{2}$ the fixed field of $\langle b\rangle$, and $E_{a b}^{2}$ the fixed field of $\langle a b\rangle$. Then for every odd prime $p$,

$$
K_{2}\left(E^{4} / E\right)(p) \cong K_{2}\left(E_{a}^{2} / E\right)(p) \times K_{2}\left(E_{b}^{2} / E\right)(p) \times K_{2}\left(E_{a b}^{2} / E\right)(p),
$$

and

$$
\left|K_{2}\left(\mathcal{O}_{E^{4}}\right)\right|\left|K_{2}\left(\mathcal{O}_{E}\right)\right|^{2}={ }_{p}\left|K_{2}\left(\mathcal{O}_{E_{a}^{2}}\right)\right|\left|K_{2}\left(\mathcal{O}_{E_{b}^{2}}\right)\right|\left|K_{2}\left(\mathcal{O}_{E_{a b}^{2}}\right)\right| .
$$

Let $E^{2^{n}} / E$ be a Galois extension with Galois group $D_{2^{n}}$. In order to get the main theorem, we give the following basic information about the dihedral group $D_{2^{n}}$.

For every $\sigma^{i} \tau \in D_{2^{n}}\left(0 \leq i \leq 2^{n-1}-1, i\right.$ an integer $)$, we have $\left(\sigma^{i} \tau\right)^{2}=$ $\sigma^{i}\left(\tau \sigma^{i} \tau\right)=\sigma^{i} \sigma^{-i}=1$, i.e., $\sigma^{i} \tau$ is of order 2. Furthermore, $\left\langle\sigma^{i} \tau\right\rangle$ and $\left\langle\sigma^{j} \tau\right\rangle$ are conjugate subgroups iff $2 \mid i+j$. Therefore, the non-trivial subgroups of $D_{2^{n}}$ and the corresponding fixed fields are as follows:

- $2^{n-1}+1$ subgroups of order $2:\left\langle\sigma^{2^{n-2}}\right\rangle$ and $\left\langle\sigma^{i} \tau\right\rangle\left(0 \leq i \leq 2^{n-1}-1, i\right.$ an integer). The corresponding fixed fields are respectively $E^{2^{n-1}}$ and $E_{i}^{2^{n-1}}$. Moreover, $\left\langle\sigma^{2 i} \tau\right\rangle\left(0 \leq 2 i \leq 2^{n-1}-2\right)$ are conjugate subgroups, and $\left\langle\sigma^{2 i+1} \tau\right\rangle\left(1 \leq 2 i+1 \leq 2^{n-1}-1\right)$ are conjugate subgroups.

- $2^{n-2}+1$ subgroups of order $4:\left\langle\sigma^{2^{n-3}}\right\rangle$ and $\left\langle\sigma^{2^{n-2}}, \sigma^{i} \tau\right\rangle(0 \leq i \leq$ $2^{n-2}-1, i$ an integer $)$, where $\left\langle\sigma^{2^{n-3}}\right\rangle$ is a cyclic group of order 4 , and every subgroup $\left\langle\sigma^{2^{n-2}}, \sigma^{i} \tau\right\rangle$ is isomorphic to $V_{4}$. The corresponding fixed fields are respectively $E^{2^{n-2}}$ and $E_{i}^{2^{n-2}}$.

- $2^{n-m}+1$ subgroups of order $2^{m}(3 \leq m \leq n-1):\left\langle\sigma^{2^{n-m-1}}\right\rangle$ and $\left\langle\sigma^{2^{n-m}}, \sigma^{i} \tau\right\rangle\left(0 \leq i \leq 2^{n-m}-1, i\right.$ an integer $)$, where $\left\langle\sigma^{2^{n-m-1}}\right\rangle$ is a cyclic group of order $2^{m}$, and every subgroup $\left\langle\sigma^{2^{n-m}}, \sigma^{i} \tau\right\rangle$ is isomorphic to $D_{2^{m}}$. The corresponding fixed fields are respectively $E^{2^{n-m}}$ and $E_{i}^{2^{n-m}}$.

THEOREM 1. Let $E^{2^{n}} / E$ be a Galois extension of number fields with Galois group $D_{2^{n}}, E^{2}$ the fixed field of $\langle\sigma\rangle$, and $E_{0}^{2^{n-1}}$ the fixed field of $\langle\tau\rangle$, $E_{1}^{2^{n-1}}$ the fixed field of $\langle\sigma \tau\rangle$. Then for every odd prime $p$,

$$
K_{2}\left(E^{2^{n}} / E^{2}\right)(p) \cong K_{2}\left(E_{0}^{2^{n-1}} / E\right)(p) \times K_{2}\left(E_{1}^{2^{n-1}} / E\right)(p),
$$

and

$$
\left|K_{2}\left(\mathcal{O}_{E^{2^{n}}}\right)\right|\left|K_{2}\left(\mathcal{O}_{E}\right)\right|^{2}={ }_{p}\left|K_{2}\left(\mathcal{O}_{E^{2}}\right)\right|\left|K_{2}\left(\mathcal{O}_{E_{0}^{2^{n-1}}}\right)\right|\left|K_{2}\left(\mathcal{O}_{E_{1}^{2^{n-1}}}\right)\right| .
$$

Proof. To prove (2.1), we will construct a map

$$
\varphi: K_{2}\left(E^{2^{n}} / E^{2}\right)(p) \rightarrow K_{2}\left(E_{0}^{2^{n-1}} / E\right)(p) \times K_{2}\left(E_{1}^{2^{n-1}} / E\right)(p),
$$

and prove that it is an isomorphism. 
From $\operatorname{tr}_{E^{2 n} / E}=\operatorname{tr}_{E_{i}^{2^{n-1}} / E} \circ \operatorname{tr}_{E^{2^{n}} / E_{i}^{2^{n-1}}}=\operatorname{tr}_{E^{2} / E} \circ \operatorname{tr}_{E^{2^{n}} / E^{2}}$, we get

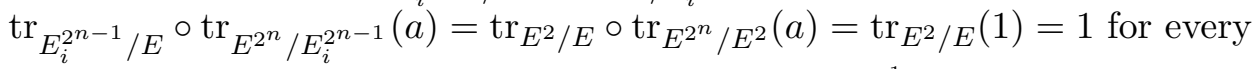
$a \in K_{2}\left(E^{2^{n}} / E^{2}\right)(p)$, hence $\operatorname{tr}_{E^{2 n} / E_{i}^{2 n-1}}(a) \in K_{2}\left(E_{i}^{2^{n-1}} / E\right)(p), i=0,1$.

Thus for every $a \in K_{2}\left(E^{2^{n}} / E^{2}\right)(p)$ we can define

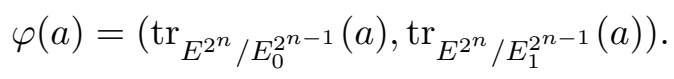

Obviously, $\varphi$ is a homomorphism.

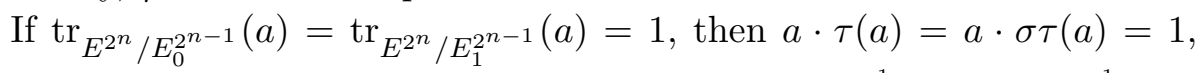
hence $\sigma(a)=a$, so $j \circ \operatorname{tr}_{E^{2^{n}} / E^{2}}(a)=a \cdot \sigma(a) \cdot \sigma^{2}(a) \cdots \sigma^{2^{n-1}-1}(a)=a^{2^{n-1}}=1$. This implies $a=1$ since $a \in K_{2}\left(E^{2^{n}} / E^{2}\right)(p)$. So $\varphi$ is injective.

For every $b \in K_{2}\left(E_{0}^{2^{n-1}} / E\right)(p)$, by Lemma 3 , there exists $c \in K_{2}\left(O_{E^{2 n}}\right)(p)$ such that

$$
b=j \circ \operatorname{tr}_{E^{2^{n}} / E_{0}^{2^{n-1}}}(c)=N_{E^{2^{n}} / E_{0}^{2^{n-1}}}(c)=c \cdot \tau(c)
$$

then

$$
\begin{aligned}
N_{E^{2^{n}} / E}(c) & =j \circ \operatorname{tr}_{E^{2^{n}} / E}(c) \\
& =j \circ \operatorname{tr}_{E_{0}^{2^{n-1}} / E} \circ \operatorname{tr}_{E^{2^{n}} / E_{0}^{2^{n-1}}}(c)=j \circ \operatorname{tr}_{E_{0}^{2^{n-1}} / E}(b)=1 .
\end{aligned}
$$

Thus

$$
j \circ \operatorname{tr}_{E^{2^{n}} / E^{2}}(b)=j \circ \operatorname{tr}_{E^{2^{n}} / E^{2}}(c \cdot \tau(c))=N_{E^{2^{n}} / E}(c)=1 .
$$

Hence $b \in K_{2}\left(E^{2^{n}} / E^{2}\right)(p)$, so $K_{2}\left(E_{0}^{2^{n-1}} / E\right)(p)$ can be considered as a subgroup of $K_{2}\left(E^{2^{n}} / E^{2}\right)(p)$. Similarly, $K_{2}\left(E_{1}^{2^{n-1}} / E\right)(p)$ can also be considered as a subgroup of $K_{2}\left(E^{2^{n}} / E^{2}\right)(p)$.

If $d \in K_{2}\left(E_{0}^{2^{n-1}} / E\right)(p) \cap K_{2}\left(E_{1}^{2^{n-1}} / E\right)(p)$, it is obvious that $d$ is fixed by $\tau$ and by $\sigma \tau$ then it is fixed by $\sigma$. Since $d \in K_{2}\left(E^{2^{n}} / E^{2}\right)(p)$, we have $\operatorname{tr}_{E^{2^{n}} / E^{2}}(d)=d^{2^{n-1}}=1$. So $d=1$, i.e.,

$$
K_{2}\left(E_{0}^{2^{n-1}} / E\right)(p) \cap K_{2}\left(E_{1}^{2^{n-1}} / E\right)(p)=1 .
$$

Thus, we have proved (2.1). By (2.1), we have

$$
\left|K_{2}\left(E^{2^{n}} / E^{2}\right)(p)\right|=\left|K_{2}\left(E_{0}^{2^{n-1}} / E\right)(p)\right|\left|K_{2}\left(E_{1}^{2^{n-1}} / E\right)(p)\right| .
$$

By Lemma 1, we conclude that

$$
\begin{aligned}
\left|K_{2}\left(\mathcal{O}_{E^{2^{n}}}\right)\right| & ={ }_{p}\left|K_{2}\left(E^{2^{n}} / E^{2}\right)\right|\left|K_{2}\left(\mathcal{O}_{E^{2}}\right)\right|, \\
\left|K_{2}\left(\mathcal{O}_{E_{i}^{2^{n-1}}}\right)\right| & ={ }_{p}\left|K_{2}\left(E_{i}^{2^{n-1}} / E\right)\right|\left|K_{2}\left(\mathcal{O}_{E}\right)\right|, \quad i=1,2 .
\end{aligned}
$$

Substituting this in (2.3) proves (2.2).

TheOREM 2. Let $E^{2^{n}} / E$ be a Galois extension of number fields with Galois group $D_{2^{n}}$, its subgroups and the corresponding fixed fields as stated 
above. Then for every odd prime $p$ and every $m \in \mathbb{Z}, 0 \leq m \leq n-2$, we have

$$
\begin{aligned}
\left|K_{2}\left(\mathcal{O}_{E^{2 n-m}}\right)\right|\left|K_{2}\left(\mathcal{O}_{E}\right)\right|^{2} & \\
& ={ }_{p}\left|K_{2}\left(\mathcal{O}_{E^{2}}\right)\right|\left|K_{2}\left(\mathcal{O}_{E_{0}^{2^{n-m-1}}}\right)\right|\left|K_{2}\left(\mathcal{O}_{E_{1}^{2^{n-m}-1}}\right)\right| .
\end{aligned}
$$

Proof. By Theorem 1, we have proved (2.4) in the case $m=0$. Next, we will prove it for $1 \leq m \leq n-2$.

Every subgroup $\left\langle\sigma^{2^{n-m-1}}\right\rangle$ is a normal subgroup of $D_{2^{n}}$, and the corresponding fixed field is $E^{2^{n-m}}$. Since $E^{2^{n}} / E$ is a Galois extension, by Galois theory $E^{2^{n-m}} / E$ is a Galois extension and $\operatorname{Gal}\left(E^{2^{n-m}} / E\right) \cong D_{2^{n}} /\left\langle\sigma^{2^{n-m-1}}\right\rangle$. Then

$$
\begin{aligned}
\operatorname{Gal}\left(E^{2^{n-m}} / E\right) & \cong D_{2^{n-m}}, \quad 1 \leq m \leq n-3, \\
\operatorname{Gal}\left(E^{4} / E\right) & \cong V_{4} .
\end{aligned}
$$

By (2.5) and Theorem 1, we get (2.4) in the case $1 \leq m \leq n-3$. By (2.6) and Lemma 4 , we get (2.4) in the case $m=n-2$. The proof is complete.

THEOREM 3. Let $E^{2^{n}} / E$ be a Galois extension of number fields with Galois group $D_{2^{n}}$, its subgroups and the corresponding fixed fields as stated above. Then for every odd prime $p$ and every $m \in \mathbb{Z}, 2 \leq m \leq n-1$, we have

$$
\begin{aligned}
& \left|K_{2}\left(O_{E^{2^{n}}}\right)\right|\left|K_{2}\left(O_{E_{0}^{2^{n-m}}}\right)\right|^{2}={ }_{p}\left|K_{2}\left(O_{E^{2^{n-m+1}}}\right)\right|\left|K_{2}\left(O_{E_{0}^{2^{n-1}}}\right)\right|^{2}, \\
& \left|K_{2}\left(O_{E^{2^{n}}}\right)\right|\left|K_{2}\left(O_{E_{1}^{2^{n-m}}}\right)\right|^{2}={ }_{p}\left|K_{2}\left(O_{E^{2^{n-m+1}}}\right)\right|\left|K_{2}\left(O_{E_{1}^{2^{n-1}}}\right)\right|^{2},
\end{aligned}
$$

and

$$
\begin{aligned}
& \left|K_{2}\left(\mathcal{O}_{E_{i}^{2 n-m}}\right)\right| \\
& \quad=p \begin{cases}\left|K_{2}\left(\mathcal{O}_{E_{0}^{2^{n-m}}}\right)\right|, & 0 \leq i \leq 2^{n-m}-1, i \text { an even integer }, \\
\left|K_{2}\left(\mathcal{O}_{E_{1}^{2^{n-m}}}\right)\right|, & 0 \leq i \leq 2^{n-m}-1, i \text { an odd integer } .\end{cases}
\end{aligned}
$$

Proof. Since $E^{2^{n}} / E$ is a Galois extension, by Galois theory so is $E^{2^{n}} / E_{i}^{2^{n-m}}$. Moreover,

$$
\begin{aligned}
\operatorname{Gal}\left(E^{2^{n}} / E_{i}^{2^{n-2}}\right) \cong V_{4}, & 0 \leq i \leq 2^{n-2}-1 \\
\operatorname{Gal}\left(E^{2^{n}} / E_{i}^{2^{n-m}}\right) \cong D_{2^{m}}, & 3 \leq m \leq n-1,0 \leq i \leq 2^{n-m}-1 .
\end{aligned}
$$

From (2.7) and Lemma 4, we get

$$
\begin{aligned}
\left|K_{2}\left(\mathcal{O}_{E^{2^{n}}}\right)\right|\left|K_{2}\left(\mathcal{O}_{E_{i}^{2^{n-2}}}\right)\right|^{2} & \\
& ={ }_{p}\left|K_{2}\left(\mathcal{O}_{E^{2^{n-1}}}\right)\right|\left|K_{2}\left(\mathcal{O}_{E_{i}^{2^{n-1}}}\right)\right|\left|K_{2}\left(\mathcal{O}_{E_{2^{2 n-1}+i}^{2^{n-1}}}\right)\right|,
\end{aligned}
$$

where $0 \leq i \leq 2^{n-2}-1$. 
From (2.8) and Theorem 1, we get

$$
\begin{aligned}
\left|K_{2}\left(O_{E^{2^{n}}}\right)\right| & \left|K_{2}\left(O_{E_{i}^{2^{n-m}}}\right)\right|^{2} \\
& ={ }_{p}\left|K_{2}\left(O_{E^{2^{n-m+1}}}\right)\right|\left|K_{2}\left(O_{E_{i}^{2^{n-1}}}\right)\right|\left|K_{2}\left(O_{E_{2^{2 n-m+1}+i}^{2^{n-1}}}\right)\right|,
\end{aligned}
$$

where $3 \leq m \leq n-1,0 \leq i \leq 2^{n-m}-1$.

Therefore, for $2 \leq m \leq n-1$ and $0 \leq i \leq 2^{n-m}-1$, we have

$$
\begin{aligned}
\left|K_{2}\left(\mathcal{O}_{E^{2^{n}}}\right)\right| & \left|K_{2}\left(\mathcal{O}_{E_{i}^{2^{n-m}}}\right)\right|^{2} \\
& ={ }_{p}\left|K_{2}\left(\mathcal{O}_{E^{2^{n-m+1}}}\right)\right|\left|K_{2}\left(\mathcal{O}_{E_{i}^{2^{n-1}}}\right)\right|\left|K_{2}\left(\mathcal{O}_{E_{2^{n-m+1}+i}^{2^{n-1}}}\right)\right| .
\end{aligned}
$$

Since $\langle\tau\rangle,\left\langle\sigma^{2} \tau\right\rangle, \ldots,\left\langle\sigma^{2^{n-1}-2} \tau\right\rangle$ are conjugate subgroups, we conclude that $K_{2}\left(\mathcal{O}_{E_{0}^{2 n-1}}\right)(p), K_{2}\left(\mathcal{O}_{E_{2}^{2 n-1}}\right)(p), \ldots, K_{2}\left(\mathcal{O}_{E_{2^{n-1}-2}^{2 n-1}}\right)(p)$ are all isomorphic, so

$$
\left|K_{2}\left(\mathcal{O}_{E_{0}^{2 n-1}}\right)(p)\right|=\left|K_{2}\left(\mathcal{O}_{E_{2}^{2^{n-1}}}\right)(p)\right|=\cdots=\left|K_{2}\left(\mathcal{O}_{E_{2^{2-1}-2}^{2^{n-1}}}\right)(p)\right| .
$$

Similarly,

$$
\left|K_{2}\left(\mathcal{O}_{E_{1}^{2 n-1}}\right)(p)\right|=\left|K_{2}\left(\mathcal{O}_{E_{3}^{2^{n-1}}}\right)(p)\right|=\cdots=\left|K_{2}\left(\mathcal{O}_{E_{2^{n-1}-1}^{2^{n-1}}}\right)(p)\right| .
$$

Hence, when $i$ is an even integer, we have

$$
\left|K_{2}\left(O_{E^{2^{n}}}\right)\right|\left|K_{2}\left(O_{E_{i}^{2^{n-m}}}\right)\right|^{2}={ }_{p}\left|K_{2}\left(O_{E^{2^{n-m+1}}}\right)\right|\left|K_{2}\left(O_{E_{0}^{2^{n-1}}}\right)\right|^{2} .
$$

When $i$ is an odd integer, we have

$$
\left|K_{2}\left(O_{E^{2^{n}}}\right)\right|\left|K_{2}\left(O_{E_{i}^{2^{n-m}}}\right)\right|^{2}={ }_{p}\left|K_{2}\left(O_{E^{2^{n-m+1}}}\right)\right|\left|K_{2}\left(O_{E_{1}^{2^{n-1}}}\right)\right|^{2} .
$$

So the theorem is proved.

3. Applications. Let $E^{16} / E$ be a Galois extension of number fields with Galois group $D_{16}=\left\langle\sigma, \tau \mid \sigma^{8}=1, \tau^{2}=1, \tau \sigma \tau^{-1}=\sigma^{-1}\right\rangle$. Its non-trivial subgroups and the corresponding fixed fields are as follows:

- 9 subgroups of order $2:\left\{1, \sigma^{4}\right\},\left\{1, \sigma^{i} \tau\right\}(0 \leq i \leq 7)$. The corresponding fixed fields are respectively $E^{8}, E_{i}^{8}(0 \leq i \leq 7)$. Furthermore, $\{1, \tau\},\left\{1, \sigma^{2} \tau\right\},\left\{1, \sigma^{4} \tau\right\}$ and $\left\{1, \sigma^{6} \tau\right\}$ are conjugate subgroups, so $E_{0}^{8}$, $E_{2}^{8}, E_{4}^{8}$ and $E_{6}^{8}$ are isomorphic subfields. Similarly, $\{1, \sigma \tau\},\left\{1, \sigma^{3} \tau\right\}$, $\left\{1, \sigma^{5} \tau\right\}$ and $\left\{1, \sigma^{7} \tau\right\}$ are conjugate subgroups, so $E_{1}^{8}, E_{3}^{8}, E_{5}^{8}$ and $E_{7}^{8}$ are isomorphic subfields.

- 5 subgroups of order 4: $\left\{1, \sigma^{2}, \sigma^{4}, \sigma^{6}\right\},\left\{1, \sigma^{4}, \tau, \sigma^{4} \tau\right\},\left\{1, \sigma^{4}, \sigma \tau, \sigma^{5} \tau\right\}$, $\left\{1, \sigma^{4}, \sigma^{2} \tau, \sigma^{6} \tau\right\}$ and $\left\{1, \sigma^{4}, \sigma^{3} \tau, \sigma^{7} \tau\right\}$. The corresponding fixed fields are respectively $E^{4}, E_{0}^{4}, E_{1}^{4}, E_{2}^{4}$ and $E_{3}^{4}$.

- 3 subgroups of order 8: $\left\{1, \sigma, \sigma^{2}, \sigma^{3}, \sigma^{4}, \sigma^{5}, \sigma^{6}, \sigma^{7}\right\},\left\{1, \sigma^{2}, \sigma^{4}, \sigma^{6}, \tau\right.$, $\left.\sigma^{2} \tau, \sigma^{4} \tau, \sigma^{6} \tau\right\}$ and $\left\{1, \sigma^{2}, \sigma^{4}, \sigma^{6}, \sigma \tau, \sigma^{3} \tau, \sigma^{5} \tau, \sigma^{7} \tau\right\}$. The corresponding fixed fields are respectively $E^{2}, E_{0}^{2}$ and $E_{1}^{2}$. 
Proposition 1. Let $E^{16} / E$ be a Galois extension of number fields with Galois group $D_{16}$, its subgroups and the corresponding fixed fields as stated above. Then for every odd prime $p$, we have

$$
\begin{aligned}
\left|K_{2}\left(\mathcal{O}_{E^{16}}\right)\right|\left|K_{2}\left(\mathcal{O}_{E}\right)\right|^{2} & ={ }_{p}\left|K_{2}\left(\mathcal{O}_{E^{2}}\right)\right|\left|K_{2}\left(\mathcal{O}_{E_{0}^{8}}\right)\right|\left|K_{2}\left(\mathcal{O}_{E_{1}^{8}}\right)\right|, \\
\left|K_{2}\left(\mathcal{O}_{E^{8}}\right)\right|\left|K_{2}\left(\mathcal{O}_{E}\right)\right|^{2} & ={ }_{p}\left|K_{2}\left(\mathcal{O}_{E^{2}}\right)\right|\left|K_{2}\left(\mathcal{O}_{E_{0}^{4}}\right)\right|\left|K_{2}\left(\mathcal{O}_{E_{1}^{4}}\right)\right|, \\
\left|K_{2}\left(\mathcal{O}_{E^{4}}\right)\right|\left|K_{2}\left(\mathcal{O}_{E}\right)\right|^{2} & ={ }_{p}\left|K_{2}\left(\mathcal{O}_{E^{2}}\right)\right|\left|K_{2}\left(\mathcal{O}_{E_{0}^{2}}\right)\right|\left|K_{2}\left(\mathcal{O}_{E_{1}^{2}}\right)\right|, \\
\left|K_{2}\left(\mathcal{O}_{E^{16}}\right)\right|\left|K_{2}\left(\mathcal{O}_{E_{0}^{4}}\right)\right|^{2} & ={ }_{p}\left|K_{2}\left(\mathcal{O}_{E^{8}}\right)\right|\left|K_{2}\left(\mathcal{O}_{E_{0}^{8}}\right)\right|^{2}, \\
\left|K_{2}\left(\mathcal{O}_{E^{16}}\right)\right|\left|K_{2}\left(\mathcal{O}_{E_{1}^{4}}\right)\right|^{2} & ={ }_{p}\left|K_{2}\left(\mathcal{O}_{E^{8}}\right)\right|\left|K_{2}\left(\mathcal{O}_{E_{1}^{8}}\right)\right|^{2}, \\
\left|K_{2}\left(\mathcal{O}_{E_{0}^{4}}\right)\right| & ={ }_{p}\left|K_{2}\left(\mathcal{O}_{E_{2}^{4}}\right)\right|, \\
\left|K_{2}\left(\mathcal{O}_{E_{1}^{4}}\right)\right| & ={ }_{p}\left|K_{2}\left(\mathcal{O}_{E_{3}^{4}}\right)\right|, \\
\left|K_{2}\left(\mathcal{O}_{E^{16}}\right)\right|\left|K_{2}\left(\mathcal{O}_{E_{0}^{2}}\right)\right|^{2} & ={ }_{p}\left|K_{2}\left(\mathcal{O}_{E^{4}}\right)\right|\left|K_{2}\left(\mathcal{O}_{E_{0}^{8}}\right)\right|^{2}, \\
\left|K_{2}\left(\mathcal{O}_{E^{16}}\right)\right|\left|K_{2}\left(\mathcal{O}_{E_{1}^{2}}\right)\right|^{2} & ={ }_{p}\left|K_{2}\left(\mathcal{O}_{E^{4}}\right)\right|\left|K_{2}\left(\mathcal{O}_{E_{1}^{8}}\right)\right|^{2}, \\
\left|K_{2}\left(\mathcal{O}_{E^{8}}\right)\right|\left|K_{2}\left(\mathcal{O}_{E_{0}^{2}}\right)\right|^{2} & ={ }_{p}\left|K_{2}\left(\mathcal{O}_{E^{4}}\right)\right|\left|K_{2}\left(\mathcal{O}_{E_{0}^{4}}\right)\right|^{2}, \\
\left|K_{2}\left(\mathcal{O}_{E^{8}}\right)\right|\left|K_{2}\left(\mathcal{O}_{E_{1}^{2}}\right)\right|^{2} & ={ }_{p}\left|K_{2}\left(\mathcal{O}_{E^{4}}\right)\right|\left|K_{2}\left(\mathcal{O}_{E_{1}^{4}}\right)\right|^{2} .
\end{aligned}
$$

Proof. The formulae (3.1)-(3.9) follow at once from Theorems 2 and 3. By Galois theory, $E^{8} / E_{0}^{2}$ is a Galois extension with Galois group $V_{4}$; its three subextensions are $E^{4} / E_{0}^{2}, E_{0}^{4} / E_{0}^{2}$ and $E_{2}^{4} / E_{0}^{2}$. By Lemma 4 , we get $\left|K_{2}\left(O_{E^{8}}\right)\right|\left|K_{2}\left(O_{E_{0}^{2}}\right)\right|^{2}={ }_{p}\left|K_{2}\left(O_{E^{4}}\right)\right|\left|K_{2}\left(O_{E_{0}^{4}}\right)\right|\left|K_{2}\left(O_{E_{2}^{4}}\right)\right|$. Hence, we get (3.10) by (3.6). Similarly, we get (3.11) from (3.7).

ExAmple. Let $\mathbb{Q}^{16}=\mathbb{Q}(i, \sqrt[8]{2} \sqrt{2+\sqrt{2}})$. It is easy to verify that $\operatorname{Gal}\left(\mathbb{Q}^{16} / \mathbb{Q}\right)=D_{16}$, where

$$
\begin{array}{ll}
\sigma(i)=i, & \sigma(\sqrt[8]{2} \sqrt{2+\sqrt{2}})=\sqrt[8]{2} \sqrt{2-\sqrt{2}} \zeta_{8}, \\
\tau(i)=-i, & \tau(\sqrt[8]{2} \sqrt{2+\sqrt{2}})=\sqrt[8]{2} \sqrt{2+\sqrt{2}} .
\end{array}
$$

Furthermore,

$$
\begin{aligned}
& \mathbb{Q}^{8}=\mathbb{Q}(i, \sqrt[4]{2}), \quad \mathbb{Q}_{0}^{8}=\mathbb{Q}(\sqrt[8]{2} \sqrt{2+\sqrt{2}}), \\
& \mathbb{Q}_{1}^{8}=\mathbb{Q}\left(\sqrt[8]{2} \sqrt{2+\sqrt{2}}+\sqrt[8]{2} \sqrt{2-\sqrt{2}} \zeta_{8}\right), \quad \mathbb{Q}_{2}^{8}=\mathbb{Q}((1+i) \sqrt[8]{2} \sqrt{2+\sqrt{2}}), \\
& \mathbb{Q}_{3}^{8}=\mathbb{Q}\left(\sqrt[8]{2} \sqrt{2+\sqrt{2}}+\sqrt[8]{2} \sqrt{2-\sqrt{2}} \zeta_{8}^{3}\right), \\
& \mathbb{Q}_{4}^{8}=\mathbb{Q}(i \sqrt[8]{2} \sqrt{2+\sqrt{2}}), \quad \mathbb{Q}_{5}^{8}=\mathbb{Q}\left(\sqrt[8]{2} \sqrt{2+\sqrt{2}}+\sqrt[8]{2} \sqrt{2-\sqrt{2}} \zeta_{8}^{5}\right),
\end{aligned}
$$


$\mathbb{Q}_{6}^{8}=\mathbb{Q}((1-i) \sqrt[8]{2} \sqrt{2+\sqrt{2}}), \quad \mathbb{Q}_{7}^{8}=\mathbb{Q}\left(\sqrt[8]{2} \sqrt{2+\sqrt{2}}+\sqrt[8]{2} \sqrt{2-\sqrt{2}} \zeta_{8}^{7}\right)$,

$\mathbb{Q}^{4}=\mathbb{Q}(i, \sqrt{2}), \quad \mathbb{Q}_{0}^{4}=\mathbb{Q}(\sqrt[4]{2}), \quad \mathbb{Q}_{1}^{4}=\mathbb{Q}((1+i) \sqrt[4]{2}), \quad \mathbb{Q}_{2}^{4}=\mathbb{Q}(i \sqrt[4]{2})$,

$\mathbb{Q}_{3}^{4}=\mathbb{Q}((1-i) \sqrt[4]{2})$,

$\mathbb{Q}^{2}=\mathbb{Q}(\sqrt{-1}), \quad \mathbb{Q}_{0}^{2}=\mathbb{Q}(\sqrt{2}), \quad \mathbb{Q}_{1}^{2}=\mathbb{Q}(\sqrt{-2})$.

For every odd prime $p$, we know that $K_{2}\left(\mathcal{O}_{\mathbb{Q}^{2}}\right)(p)=K_{2}\left(\mathcal{O}_{\mathbb{Q}_{0}^{2}}\right)(p)=$ $K_{2}\left(\mathcal{O}_{\mathbb{Q}_{1}^{2}}\right)(p)=K_{2}\left(\mathcal{O}_{\mathbb{Q}^{4}}\right)(p)=1$. By Proposition 1 , we have

$$
\begin{gathered}
\left|K_{2}\left(\mathcal{O}_{\mathbb{Q}_{i}^{4}}\right)\right|={ }_{p}\left|K_{2}\left(\mathcal{O}_{\mathbb{Q}_{j}^{4}}\right)\right|, \quad 0 \leq i, j \leq 3, \\
\left|K_{2}\left(\mathcal{O}_{\mathbb{Q}_{i}^{8}}\right)\right|={ }_{p}\left|K_{2}\left(\mathcal{O}_{\mathbb{Q}_{j}^{8}}\right)\right|, \quad 0 \leq i, j \leq 7, \\
\left|K_{2}\left(\mathcal{O}_{\mathbb{Q}^{8}}\right)\right|={ }_{p}\left|K_{2}\left(\mathcal{O}_{\mathbb{Q}_{0}^{4}}\right)\right|^{2}={ }_{p}\left|K_{2}\left(\mathcal{O}_{\mathbb{Q}_{1}^{4}}\right)\right|^{2}, \\
\left|K_{2}\left(\mathcal{O}_{\mathbb{Q}^{16}}\right)\right|={ }_{p}\left|K_{2}\left(\mathcal{O}_{\mathbb{Q}_{0}^{8}}\right)\right|^{2}={ }_{p}\left|K_{2}\left(\mathcal{O}_{\mathbb{Q}_{1}^{8}}\right)\right|^{2} .
\end{gathered}
$$

Acknowledgements. The authors are grateful to the anonymous referees for their very careful reading of the paper and useful comments. The research work of H. Y. Zhou is supported by Post-Doctoral Funds of Jiangsu (1201065C).

\section{References}

[1] J. Browkin, On the p-rank of the tame kernel of algebraic number fields, J. Reine Angew. Math. 432 (1992), 135-149.

[2] J. Browkin, Tame kernels of cubic cyclic fields, Math. Comp. 74 (2005), 967-999.

[3] J. Browkin, Tame and wild kernels of quadratic imaginary number fields, Math. Comp. 68 (1999), 291-305.

[4] J. Browkin and H. Gangl, Tame kernels and second regulators of number fields and their subfields, submitted to the volume dedicated to Professor Aderemi O. KuKu.

[5] F. Keune, On the structure of $K_{2}$ of the ring of integers in a number field, $K$-Theory 2 (1989), 625-645.

[6] H. R. Qin, The 2-Sylow subgroup of $K_{2}\left(\mathcal{O}_{F}\right)$ for number fields $F$, J. Algebra 284 (2005), 494-519.

[7] H. R. Qin, The 2-Sylow subgroups of the tame kernel of imaginary quadratic fields, Acta Arith. 69 (1995), 153-169.

[8] H. R. Qin, The 4-rank of $K_{2}\left(O_{F}\right)$ for real quadratic fields, Acta Arith. 72 (1995), 323-333.

[9] D. Quillen, Finite generation of the groups $K_{i}$ of rings of algebraic integers, in: Lecture Notes in Math. 341, Springer, 1973, 179-198.

[10] C. Soulé, Groupes de Chow et K-théorie de variétés sur un corps fini, Math. Ann. 268 (1984), 317-345.

[11] J. Tate, Relation between $K_{2}$ and Galois cohomology, Invent. Math. 36 (1976), 257274.

[12] H. Y. Zhou, Odd parts of tame kernels of dihedral extensions, Acta Arith. 156 (2012), $341-349$. 
[13] H. Y. Zhou, The tame kernel of multiquadratic number fields, Comm. Algebra 37 (2009), 630-638.

[14] H. Y. Zhou, Tame kernels of cubic cyclic fields, Acta Arith. 124 (2006), 293-313.

Qianqian Cui, Haiyan Zhou (corresponding author)

School of Mathematical Sciences

Nanjing Normal University

Nanjing, 210023

P.R. China

E-mail: cuiqianqiande@126.com

haiyanxiaodong@gmail.com

Received February 21, 2013 
